\title{
Searchlight CT: A new reconstruction method for collimated X-ray tomography
}

\author{
Robert Azencott \\ 608 PGH, University of \\ Houston \\ 4800 Calhoun Road \\ Houston, Texas 77204 \\ razencot@math.uh.edu
Anando Sen
648 PGH, University of Houston
4800 Calhoun Road
Houston, Texas 77204
anando@math.uh.edu

\author{
Bernhard G. Bodmann \\ 604 PGH, University of \\ Houston \\ 4800 Calhoun Road \\ Houston, Texas 77204 \\ bgb@math.uh.edu
}

King C. Li, M.D.

The Methodist Hospital

Research Institute

6565 Fannin Street

Houston, Texas 77030

kli@tmhs.org

\author{
Demetrio Labate \\ 694 PGH, University of \\ Houston \\ 4800 Calhoun Road \\ Houston, Texas 77204 \\ dlabate@math.uh.edu \\ Xiaobo Zhou, M.D. \\ The Methodist Hospital \\ Research Institute \\ 6565 Fannin Street \\ Houston, Texas 77030 \\ xzhou@tmhs.org
}

\begin{abstract}
The method presented in this paper aims to reduce the overall radiation exposure from X-ray CT scans when only the reconstruction of a region of interest is required. To achieve this goal, the Searchlight CT algorithm restricts the acquisition to X-rays passing mainly through the region of interest. The algorithm uses an iteration of the X-ray transform and of a regularized inverse, which converges rapidly and for which generic conditions of converges are provided. The performance of the Searchlight CT algorithm is illustrated on both phantom and experimental data.
\end{abstract}

\section{General Terms}

Inverse Problems

\section{Keywords}

ACM proceedings, X-ray Transform, Radon Transform, Tomography, Local Reconstruction

\section{INTRODUCTION}

The goal of this paper is to reconstruct a specifically targeted region of an unknown 3-dimensional object $F$ when only partial information of its X-ray transform is available. This is motivated by medical imaging applications since the use of collimated X-ray projections, i.e., projections acquired by restricting the irradiation to a specific region of interest inside $F$, has the effect of reducing the overall radiation dose received by $F$ during a $\mathrm{CT}$ scan.

The X-ray transform is the underlying mathematical framework for computed tomography (CT) and several other meth- ods employed to determine structural properties of objects by using projected information. Formally, the X-ray transform maps a function $F$ defined on $\mathbb{R}^{3}$ into the set of its line integrals. That is, if $F: I^{3} \rightarrow \mathbb{R}$ is a Lebesgue-integrable function on the set $I^{3}$, where $I$ is an interval of $\mathbb{R}, w \in \mathbb{R}^{3}$ and $\theta$ is a unit vector in $\mathbb{R}^{3}$, the value of the $X$-ray Transform of $F$ at $(w, \theta)$ is the line integral of $F$ over the straight line $l(w, \theta)$, through $w$ with direction $\theta$, defined by

$$
X F(w, \theta)=\int_{-\infty}^{\infty} F(w+t \theta) d t .
$$

The problem of interest consists in recovering $F$ from the values of $X F$, that is, inverting the transform. However, while it is possible to write an explicit mathematical expression for the inversion formula, it turns out that the problem of reconstructing $F$ from the values of its X-ray transform is numerically unstable, and this numerical instability becomes even more challenging if one desires to recover $F$ from an incomplete set of X-ray data [10].

In this paper, we are interested in a special instance of reconstruction from incomplete data, where the goal is the reconstruction of a specific Region of Interest, denoted $S$, inside an unknown 3-dimensional density function $F$, using only the X-rays passing through $S$. This problem is motivated by medical diagnostic applications where it is necessary to frequently and regularly monitor a "small" region inside the human body (e.g., to verify the evolution of a tumor), and, at the same time, it is important to reduce the overall radiation exposure of the patient.

As expected, naive reconstruction attempts introduce many undesirable artifacts. Here, we introduce a new numerical algorithm for the accurate density reconstruction on a Region of Interest $S$ which only requires an acquisition with X-rays collimated on $S$. The algorithm is based on an iterative procedure, for which we are able to provide generic convergence conditions. 


\section{COLLIMATED RECONSTRUCTION}

The classical formula for the inversion of the X-ray transform is based on the Fourier Slice Theorem [9] which leads to the so-called filtered back-projection. Specifically, we have

$$
\begin{aligned}
F(x, y, z) & =X^{-1}(X F)(x, y, z) \\
& =\int_{\mathbb{R}^{3}} \mathcal{F}_{2}\left(X_{\theta} F\right)(\xi) e^{i((x, y, z) \cdot \xi)} d \xi
\end{aligned}
$$

where $X_{\theta} F(w)=X F(w, \theta), \mathcal{F}_{2}$ denotes the 2D Fourier transform on a plane $h(\theta(\xi))$ with normal vector $\theta(\xi)$, and $\theta(\xi)$ is any vector orthogonal to $\xi$

We are interested in reconstructing a spherical region $S$ contained inside a 3-dimensional $F \subset I^{3}$, under the assumption that mainly the X-rays passing through $S$ are used in the acquisition. In view of of this, we denote the retained part of the X-ray transform as,

$$
T=\{(w, \theta): l(w, \theta) \cap S \neq \emptyset\}
$$

and $U=T^{C}$. Hence, to model the modified acquisition, we define the collimated $X$-ray transform by

$$
\widetilde{X} F(w, \theta)=\left\{\begin{array}{cc}
X F(w, \theta) & (w, \theta) \in T \\
0 & (w, \theta) \in U .
\end{array}\right.
$$

Our objective is the computation of an accurate approximation of the unknown function $F$, restricted to the region of interest $S$, when the data $\widetilde{X} F$ are given.

An important step in our Searchlight CT iterative algorithm is the (repeated) regularization of computed density function $F$ outside the region of interest $S$. To this end, we partition the complement of $S$ in $I^{3}$ into subsets $\left\{Q_{j}\right\}$, each subset of a fixed volume $\operatorname{vol}\left(Q_{j}\right)=v$, and perform local averages. We define the regularization operator $\sigma$ as

$$
\sigma F(x, y, z)=\left\{\begin{array}{cc}
F(x, y, z) & (x, y, z) \in S \\
\tau\left(F, Q_{j}\right) & (x, y, z) \in Q_{j} .
\end{array}\right.
$$

Here,

$$
\tau\left(F, Q_{j}\right)=\frac{1}{v} \iiint_{Q_{j}} F(x, y, z) d x d y d z
$$

Multiple variants of our regularization operator $\sigma$ can be used, but will not be considered here for brevity.

The Searchlight $\mathrm{CT}$ algorithm is initialized by setting the collimated data as $G=\tilde{X} F=1_{T} \cdot X F$, where $F$ is an unknown density function and the dot denotes point-wise multiplication of the functions $1_{T}$ and $X F$. We obtain the first approximation of $F$, denoted as $f_{0}$, by inverting $G$. Hence $f_{0}=X^{-1} G$, where the operator $X^{-1}$ is implemented using the standard filtered back projection by the Fourier Slice Theorem, identical to the expression in (2). The subsequent approximations $f_{n}$ of $F$ are obtained through the following iterative procedure.

For $n=1,2, \ldots$,

1. Compute $\sigma f_{n}$ as in (4).
2. Compute the standard X-ray Transform $X \sigma f_{n}$ of $\sigma f_{n}$ by (1). Separating X-rays into the two complementary sets $T$ and $U$ one obtains

$$
X \sigma f_{n}=1_{T} \cdot X \sigma f_{n}+1_{U} \cdot X \sigma f_{n}
$$

3. Replace $1_{T} \cdot X \sigma f_{n}$ by the known collimated data $G=$ $1_{T} . X F$ in the preceding formula to define $Y_{n}=G+$ $1_{U} \cdot X \sigma f_{n}$.

4. Finally compute $f_{n+1}$ by applying the X-ray Inversion formula (2) to $Y_{n}$. Hence,

$$
f_{n+1}=X^{-1} Y_{n}=X^{-1}\left[G+1_{U} \cdot X \sigma f_{n}\right]
$$

The sequence of functions $f_{n}$ generated by this algorithm converges to a function $h$ which is an approximation of the unknown function $F$, inside the region of interest $S$. This is discussed in detail in the next section.

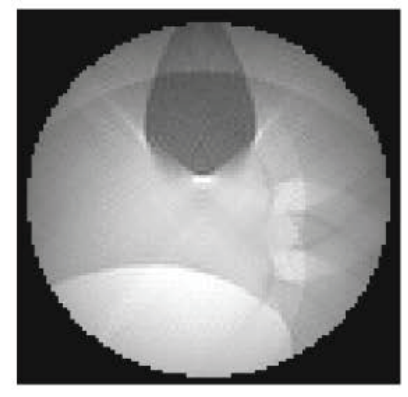

Standard Algorithm

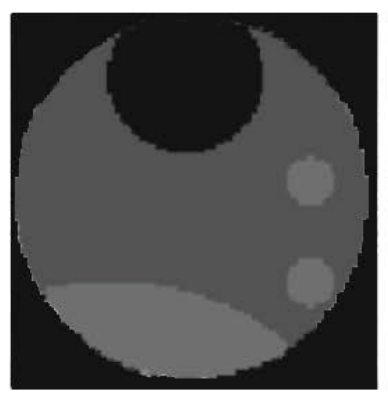

Searchlight CT

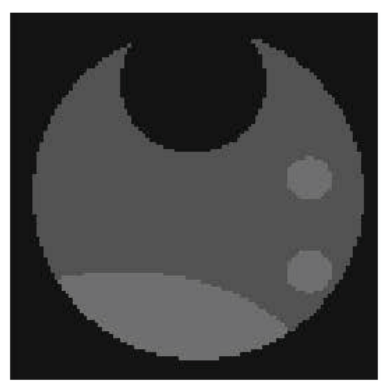

Ground Truth

Figure 1: $\mathrm{XZ}$ view of the reconstruction of a region $S$ inside a 3D Shepp-Logan Phantom using the standard Backprojection algorithm (above) and the Searchlight CT algorithm (middle). 


\section{ANALYSIS OF CONVERGENCE}

To discuss the convergence of the algorithm, we need some technical assumptions on the unknown density function $F$. Specifically, we assume that the support of $F$ is contained in a fixed closed ball $B$, and that the regularized density $\sigma F$ has good approximations by regularized versions of $F$ generated by convolving $F$ with Gaussian kernels having variances tending to 0 .

The set of X-ray source positions $P$ is taken to be disjoint from $B$ and assumed to be large enough to ensure the existence of a formal inverse $X^{-1}$ correctly defined for uncollimated data. This situation is satisfied, in particular, if $P$ is a complete sphere.

The iterative algorithm generates $f_{n+1}$ from $f_{n}$ by (5), starting from the given collimated data $G=1_{T} \cdot X F$. Since $\sigma$ is idempotent, the functions $h_{n}=\sigma f_{n}$ verify the iterative formula,

$$
h_{n+1}=\sigma X^{-1} G+\sigma X^{-1}\left(1_{U} \cdot X h_{n}\right) .
$$

Denote by $A$ the fixed function $A=\sigma X^{-1} G$ and by $M$ the linear operator which maps $h$ to $M h=\sigma X^{-1}\left(1_{U} \cdot X h\right)$. Hence, we have

$$
h_{n+1}=A+M h_{n} .
$$

which implies

$$
h_{n+1}-h_{n}=M\left(h_{n}-h_{n-1}\right) .
$$

Assuming that the density functions $F$ lies in a vector space $H$ and that the space $\sigma(H)$ can be endowed with a Banach norm such that the operator $M$ becomes a strict contraction in the Banach space $\sigma(H)$, then the iteration converges to the fixed point of the contraction. A necessary condition for convergence is that the norm of the function $F$ outside $S$ is sufficiently small. This can be verified by considering a function, which vanishes inside $S$.

Extensive numerical tests have indeed verified that the algorithm converges, under reasonable conditions on the space $H$ and the regularization operator $\sigma$, provided that the spherical region of interest $S$ is large enough, and provided that the indicator function $1_{U}$ is slightly regularized in the space of rays by convolution with a Gaussian kernel. A more rigorous analysis of the convergence is beyond this scope of this paper and will be given elsewhere.

The sequence of functions $h_{n}$ converges to a limit $h$ in $\sigma(H)$ at geometric speed, and the limit $h$ satisfies

$$
h=A+M h .
$$

Since $M$ is a strict contraction, this last equation has a unique solution in $\sigma(H)$ which is the fixed point of the iteration (7a).

Under the assumption that $F \in \sigma(H)$, we verify that $F$ satisfies the equation

$$
A+M F=\sigma X^{-1}\left(1_{T} \cdot X F\right)+\sigma X^{-1}\left(1_{U} \cdot X F\right)=F,
$$

which, by the uniqueness of $h$, implies $h=F$. Thus, for $F \in \sigma(H)$, the iterative approximations $h_{n}$ converge to the true density function $F$.
However, in general, $F \notin \sigma(H)$ and, in this situation, the iterative procedure will only produce an approximation of $F$. Since $M$ is a strict contraction, the operator $I d-M$ has a bounded inverse and hence the solution $h$ of the equation $h=A+M h$ is continuous in $A$. Thus, if $F$ and $\sigma(F)$ are sufficiently close, then the limit $h$ of $h_{n}$, as $n \rightarrow \infty$, is as close as desired to the unknown function $F$.

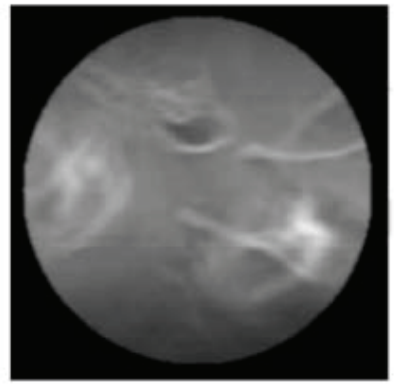

Standard Algorithm

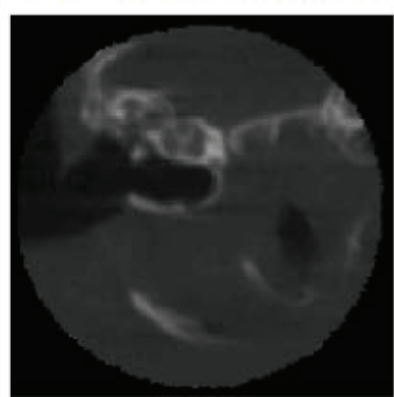

Searchlight CT

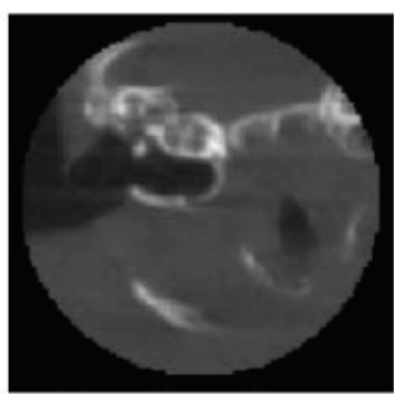

Ground Truth

Figure 2: $\mathrm{XZ}$ view of the reconstruction of a region $S$ from a biological tissue sample using the standard Backprojection algorithm (above) and the Searchlight CT algorithm (middle). The 3D biological data set was provided by Center of Biomedical Engineering, UTMB].

\section{NUMERICAL DEMONSTRATIONS}

We have tested the Searchlight CT algorithm for collimated reconstruction first on a set of 3D Shepp-Logan phantoms and next on a set of biological data. The reconstruction accuracy was evaluated by various performance measures.

We simulated the collimated acquisition when the X-ray emitting source takes arbitrary (discretized) positions on a 
fixed large sphere around the object, where the object data and X-ray transform data were discretized as follows. The simulated phantoms have been taken of size $257^{3}$ voxels. The real tissue density data were originally of size $512 \times 512 \times 768$ from which a subset of size $257^{3}$ was extracted. The regions of interest we have considered are euclidean balls $S \in I^{3}$, having arbitrary centers, and various radiuses larger than 40 voxels. Indeed, as reported below, for a given density $F \in I^{3}$, the ratio $\left\|1_{S} x F\right\|_{L_{1}} /\|F\|_{L_{1}}$ must be larger than a certain lower bound to ensure good performance of collimated reconstruction. The partition of the complement of $S$ in $I^{3}$ were chosen to be 8-voxel cubes. The size of the (simulated) planar $2 \mathrm{D}$ set of acquisition sensors was kept fixed and equal to $452 \times 370$ voxels for all source positions. The distance between the center of this planar set of sensors and the source position were also kept constant.

\section{PERFORMANCE OF SEARCHLIGHT CT}

For any voxel $v=(x, y, z)$ let $F(v)$ and $h(v)$ be the original and reconstructed densities respectively. Then the Relative Reconstruction Error, Rel is defined as,

$$
R e l=\frac{\sum_{v \in S}|F(v)-h(v)|}{\sum_{v \in S} F(v)}
$$

As a comparison, we applied the standard filtered backprojection method, which is valid for uncollimated data, and found that it always generates unacceptable inaccuracies and artifacts when applied to collimated data. For example, using a typical 3D Shepp-Logan phantom, the standard filtered back-projection method with collimated data produces a highly inaccurate reconstruction with $R e l=23.6 \%$; by contrast the Searchlight CT algorithm does significantly better with $R e l=9.5 \%$. The reconstructions are illustrated in Figure 1.

The above results were obtained using 40 iterations, which is approximately the number of iterations required for the algorithm to converge with sufficient accuracy. When the algorithm is applied to biological data, the number of iterations remains the same with similar values of Rel. This reconstruction is illustrated in Figure 2.

\subsection{Performance Criteria for Reconstruction}

The radiation dose $d(v)$ received by a voxel $v$ is defined as the number of rays passing through $v$. Let $c=\sum_{v \in I^{3}} d(v)$ be the sum of received doses over all voxels in a collimated case and $m$ be the maximal dose which is attained in the uncollimated case. We define the Radiation Exposure $E x$, as

$$
E x=\frac{c}{m} .
$$

As $E x$ increases, the reconstruction improves and hence Rel reduces, and this is illustrated in Figure 3 using the same data described above.

Another standard measure of performance we use is the Peak Signal to Noise Ratio or PSNR, which is defined

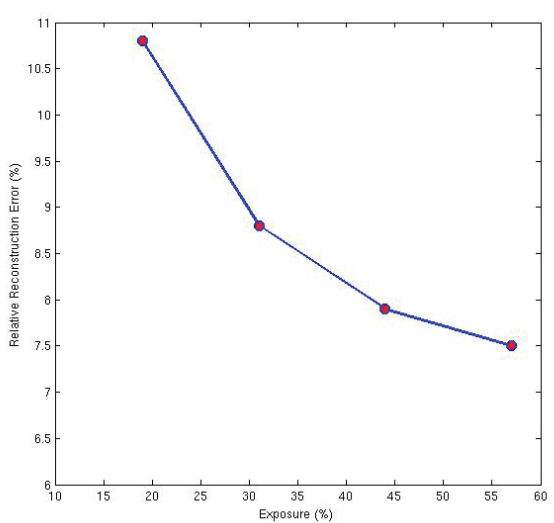

Figure 3: Graph of the Relative Reconstruction Error vs Radiation Exposure, for a typical 3D SheppLogan phantom.

as,

$$
P S N R=20 \log _{10}\left(\frac{\max (F)}{R M S E}\right)
$$

where,

$$
R M S E=\sqrt{\sum_{v \in S}(F(v)-h(v))^{2}}
$$

As stated in the previous section, the spherical region of interest $S$ must be large enough to ensure good reconstruction of collimated reconstruction. To determine which region size is large enough we introduce the notion of relative density.

The Relative Density $D$ of a region $S$ is defined as the ratio between the sum of densities over voxels on $S$ and the sum of densities over all voxels.

Table 1: Performance tests vs. radius of $S$

\begin{tabular}{|c|c|c|c|c|}
\hline$S$-radius & $D$ & $E x$ & Rel & PSNR \\
\hline 45 voxels & $3.8 \%$ & $19 \%$ & $10.3 \%$ & 58.9 \\
\hline 60 voxels & $8.5 \%$ & $31 \%$ & $8.6 \%$ & 62.2 \\
\hline 75 voxels & $15.3 \%$ & $44 \%$ & $7.6 \%$ & 63.4 \\
\hline 90 voxels & $23.8 \%$ & $57 \%$ & $7.2 \%$ & 64.0 \\
\hline
\end{tabular}

Our numerical experiments show that $D$ must be higher than $2.5 \%$ for good collimated reconstruction. Table 1 shows the values of Ex, Rel and $D$ and PSNR as a function of the radius of the region of interest $S$ for a typical 3D Shepp Logan Phantom. For a radius below 40 voxels, $D$ falls below $2.5 \%$ and the reconstruction fails. This is in agreement with our theoretical analysis of convergence criteria. The relative density $D$ increases when we increase the radius of region $S$, and simultaneously the set of rays $U$ defined above decreases, which ensures that the norm of the operator $M$ decreases.

\subsection{Comparison with Other Algorithms}

The problem of inverting the X-ray or Radon transform from limited data has received great attention in the literature, 
due to its impact in applications. However, most research so far was focused on extracting only the singular components (i.e., the egdes) of the data $[8,12,2,6,5]$. Other results, such are those in [13], can only be applied to the Radon transform in even dimensions, and even in this situation the performance of these methods is not fully satisfactory.

In particular, we compared the performance of our algorithm against to the one proposed in [11], using 2D Shepp-Logan phantoms (since the method in [11] is 2-dimensional). For a typical phantom, our Searchlight CT collimated reconstruction algorithm yields $\mathrm{Rel}=4.1 \%$ while the algorithm proposed in [11] yields Rel $=7.7 \%$. Using the standard filtered back-projection the reconstruction relative error is $R e l=11.2 \%$. Another major difference concerns the performance in terms of the exposure Ex. Using the method from [11], the exposure is $45 \%$, while our Searchlight CT algorithm yields radiation exposure $27 \%$, which is considerably smaller.

\section{FUTURE RESEARCH}

We have introduced a new reconstruction algorithm of 3D data using collimated tomography, called Searchlight CT, and its performance was successfully validated on both synthetic phantoms and biological tissue data. However, the complete spherical distribution of source positions simulated (in $2 \mathrm{D}$ and in $3 \mathrm{D}$ ) in this paper is still a fairly theoretical implementation of collimated CT acquisitions. We are currently adapting our reconstruction algorithm to the setting of spiral tomography, which is a much more practical and realistic settings in 3D tomography [15].

\section{ACKNOWLEDGMENTS}

This work was partially supported by NSF DMS 0604561 and a two year Methodist Hospital grant. We acknowledge Dr. Patrikeev and Dr. Wei, from the Center of Biomedical Engineering, University of Texas Medical Branch in Galveston, for providing the micro-CT data from which the images in Figure 2 were computed.

\section{REFERENCES}

[1] A. Averbuch and Y. Shkolnisky. 3D discrete X-ray Transform. Applied and Computational Harmonic Analysis, 17:259-276, 2004.

[2] C. Berenstein and D. Walnut. Local inversion of the radon transform in even dimensions using wavelets. In In 75 Years of Radon Transform, S. Gindikin and P. Michor, editors., pages 45-69. International Press, 1992.

[3] D. Bharkhada, H. Yu, R. Dixon, Y. Wei, J. Carr, D. Bourland, R. Hogan, and G. Wang. Demonstration of dose and scatter reduction for interior tomography. Computer Assisted Tomography, 33(6):967-72, 2009.

[4] C. Epstein. Introduction to the Mathematics of Medical Imaging, Second Edition. SIAM: Society for Industrial and Applied Mathematics, 2007.

[5] A. Faridani, D. Finch, E. Ritman, and K. Smith. Local tomography ii. SIAM Journal of Applied Mathematics, 57(4):1095-1127, 1997.

[6] A. Faridani, E. Ritman, and K. Smith. Local tomography. SIAM Journal of Applied Mathematics, 51:459-484, 1193-1198, 1992.
[7] A. Katsevich. Improved cone beam local tomography. Inverse Problems, 22:627-643, 2006.

[8] A. Katsevich and A. Ramm. Pseudolocal tomography. SIAM Journal of Applied Mathematics, 56(1):167-191, 1996.

[9] F. Natterer. The Mathematics of Computerized Tomography. SIAM: Society for Industrial and Applied Mathematics, 2001.

[10] F. Natterer and F. Wubbeling. Mathematical Methods in Image Reconstruction. SIAM: Society for Industrial and Applied Mathematics, 2001.

[11] T. Olson and J. Destefano. Wavelet localization of the radon transform. Technical Report PCS-TR93-196, Dartmouth College, Computer Science, Hanover, NH, 1993.

[12] E. Quinto. Singularities of the X-ray transform and limited data tomography in $R^{2}$. SIAM Journal of Mathematical Analysis, 24:1215-1225, 1993.

[13] F. Rashid Farrokhi, K. Ray Liu, C. Berenstein, and D. Walnut. Wavelet-based multiresolution local tomography. IEEE Transactions on Image Processing, 6(10):1412-1429, 1997.

[14] J. Yang, H. Yu, M. Jiang, and G. Wang. High-order total variation minimization for interior tomography. Inverse Problems, 26:1-29, 2010.

[15] H. Yu and G. Wang. Studies on implementation of Katsevich algorithm for spiral cone beam CT. Journal of X-Ray Science and Technology, 12(2/2004):97-116, 2004. 